\title{
Multi-level of Fidelity Multi-Disciplinary Design Optimization of Small, Solid-Propellant Launch Vehicles
}

\author{
By Jafar Roshanian, Jahangir Jodei, Mehran Mirshams, Reza Ebrahimi and Masood Mirzaee \\ MDO Laboratory, Department of Aerospace and Mechanical Engineering, K.N. Toosi University of Technology, Tehran, Iran
}

(Received July 20th, 2007)

\begin{abstract}
A new automated multi-level of fidelity Multi-Disciplinary Design Optimization (MDO) methodology has been developed at the MDO Laboratory of K.N. Toosi University of Technology. This paper explains a new design approach by formulation of developed disciplinary modules. A conceptual design for a small, solid-propellant launch vehicle was considered at two levels of fidelity structure. Low and medium level of fidelity disciplinary codes were developed and linked. Appropriate design and analysis codes were defined according to their effect on the conceptual design process. Simultaneous optimization of the launch vehicle was performed at the discipline level and system level. Propulsion, aerodynamics, structure and trajectory disciplinary codes were used. To reach the minimum launch weight, the Low LoF code first searches the whole design space to achieve the mission requirements. Then the medium LoF code receives the output of the low LoF and gives a value near the optimum launch weight with more details and higher fidelity.
\end{abstract}

Key Words: Launch Vehicles, Multi-Disciplinary Design Optimization, Solid-Propellant Rocket Motor, Fidelity, Conceptual Design

\section{Nomenclature}

$a$ : semi-major axis, shell radius

A: nozzle exit area

$d$ : nose cone diameter

$D$ : diameter

$e$ : eccentricity

$E$ : module of elasticity

$f$ : safety factor

$F$ : force, objective function

$g$ : earth gravitational constant

$h$ : thickness, height

$I_{\mathrm{sp}}$ : specific impulse

$K, k^{\prime}, k_{\mathrm{M}}$ : correction factor

$l_{y}$ : axial distance from nose to attitude control mechanism

$L$ : length

$m$ : mass

$M$ : moment

$P$ : pressure

$r$ : vehicle radius vector from earth center

$t$ : time

$T$ : thrust

$V$ : velocity

$V^{\prime}$ : required orbital velocity

$x_{\mathrm{m}}$ : axial distance from nose to center of mass

$x_{\mathrm{p}}$ : axial distance from nose to center of pressure

$X$ : axial force in body coordination system

$\left(x_{1}, y_{1}\right)$ : launch coordination system axes traveling with body center of gravity

$\left(x_{2}, y_{2}\right)$ : velocity coordination system axes

$\left(x_{\mathrm{s}}, y_{\mathrm{s}}\right)$ : launch coordination system axes
$Y$ : lateral force in body coordination system

$\alpha$ : angle of attack, motor structural factor

$\theta$ : path angle with respect to launch coordination system

$\vartheta$ : pitch angle

$\mu$ : gravitational parameter

$\sigma:$ thrust angle relative to body coordination system

$v$ : Poisson ratio

$\varphi$ : local horizon path angle

$\chi$ : angle of vehicle radius vector with respect to vertical axis of launch coordination system

Subscripts

aer: aerodynamics

c: control

cr: critical

e: exit

eng: engine, motor

eq: equivalent

f: fuel, propellant

h: height

$i$ : index of stage number

P: payload

stg: stage

$\mathrm{t}$ : termination

$\mathrm{v}$ : vacuum

$x: x$ axis

\section{Introduction}

The increasing complexity of engineering systems has sparked interest in multi-disciplinary design, analysis and optimization. Aerospace vehicle conceptual design is dominated by interactions among various traditional engineering 
disciplines. Aerodynamics, propulsion, trajectory, weights, structure and others are usually highly coupled, and complete vehicle analysis requires an iterative process with efficient method of communication among the disciplines. Progress in automation of multi-disciplinary design and analysis process has been getting faster in recent years using various analysis tools. ${ }^{1-8)}$

The two main challenges of Multi-Disciplinary Design Optimization (MDO) are computational expense and organizational complexity. In this regard, mathematical modeling and formulation of the design problem play the main role. Employing physics-based rather than empirical database driven analysis implies a greater need for computation and higher-fidelity tools. This quickly creates a combinatorial problem of computationally expensive function calls when trying to assess a reasonably large space. Several approximation techniques have been introduced to solve this problem. To incorporate different fidelity models into automatic optimization design process, Variable Complexity Modeling (VCM) has been presented and followed by several further works. ${ }^{9-17)}$ All these works address use of variable fidelity at the disciplinary level, but this study focuses on variable fidelity in MDO systems or Multi-Level of Fidelity (Multi-LoF) MDO.

However, it is (not entirely truthfully) sometimes said, that Multi-Disciplinary Analysis (MDA) is only good as the weakest link. One can build MDO systems with very impressive interfaces and sophisticated database management capabilities, but if the analysis codes are not well developed, the system may be useless or worse. ${ }^{18)}$ As a result, the contents of disciplinary codes at the conceptual design level should be harmonized with problem requirements. In this study, all disciplinary codes have been developed based on MDO requirements and problem conditions.

An approach to MDO of a multistage, Small, SolidPropellant Launch Vehicle (SSPLV) is presented by Jodei et al. ${ }^{19)}$ This paper develops a new Multi-LoF MDO structure for SSPLV using two LoF Structure. In each level, the propulsion, aerodynamics, trajectory and structure are selected as the main disciplines during conceptual design. Data flow between disciplines is addressed.

\section{Multi-LoF MDO}

\subsection{Concept}

To recognize Multi-LoF MDO, first, the concept of fidelity needs to be defined and then the purpose and structured differences between VCM and Multi-LoF should be discussed. Finally, the concept of Multi-LoF MDO structure is explained. Since the early development of simulation technology, the notion of fidelity has been an apparent issue recognized by the modeling and simulation community in properly assessing the validity and credibility of simulation results. Seven attributes were addressed as fidelity quantifications and measurement: ${ }^{20)}$ order, accuracy, precision, timeliness, error sources, consistency, and repeatability.

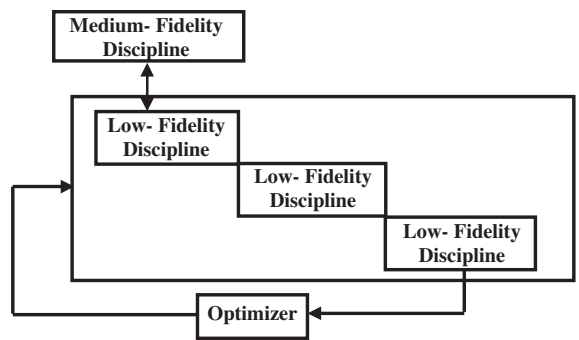

(a) VCM

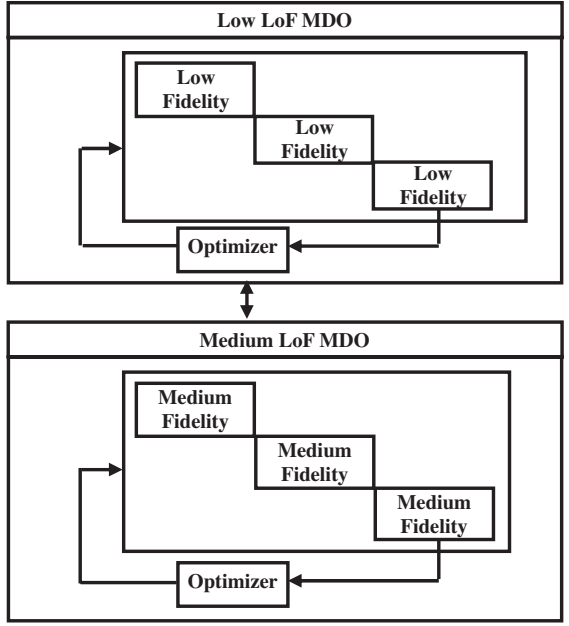

(b) Multi-LoF

Fig. 1. Differences between VCM and Multi-LoF.

Recent research into variable fidelity in MDO has focused on VCM, formulating surrogate single disciplines with different LoF into a single MDO. The Multi-LoF concept recommends miscellaneous fidelity in the whole MDO formulation. Figure 1 shows the difference between the VCM and Multi-LoF concepts. A MDO problem with three disciplines is considered in Fig. 1(a). The new concept Multi-LoF MDO presented here, can be configured with two or more LoF MDO structures (i.e. low and medium LoF-Fig. 1(b)).

The fidelity of the MDO structure depends on the fidelity of the organizing disciplines. The low LoF MDO consists of disciplines with low-fidelity codes, and the high LoF MDO structure consists of high-fidelity codes. The Multi-LoF can be applied to all MDO formulations, including single layer (i.e. Multi-Disciplinary Feasible (MDF) and IndividualDisciplinary Feasible (IDF), or multi layer, such as Collaborative Optimization (CO)).

The Multi-LoF MDO should be analyzed from two different viewpoints: procedural, and computational. In the procedural viewpoint, conceptual design of conventional aerospace system begins with a few data followed by an interactive process to increase knowledge of system design parameters. As system design progresses to deeper levels, the complexity of analysis tools and the fidelity of models with respect to reality increase. In practice, the LoF of models changes with use of analytical, empirical, statistical, and numerical methods. In fact, these methods change with fidelity attributes, such as order, accuracy, timeliness, precision (resolution), error source, consistency, and repeatability. 
Table 1. Different LoF in SSPLV conceptual design.

\begin{tabular}{|c|c|c|c|}
\hline \multirow{2}{*}{ Disciplines } & \multicolumn{3}{|c|}{$\mathrm{LoF}$} \\
\hline & Low & Medium & High \\
\hline Propulsion & $\begin{array}{l}\text { - Representative SRM installed in } \\
\text { representative configuration based } \\
\text { on available technologies }\end{array}$ & $\begin{array}{l}\text { - Representative SRM installed } \\
\text { in like configuration } \\
\text {-Analytical design relationship }\end{array}$ & $\begin{array}{l}\text {-Optimized parametric SRM installed } \\
\text { analytically } \\
\text {-Major subsystems designed and feedback } \\
\text { to correct basic design variables }\end{array}$ \\
\hline Aerodynamics & $\begin{array}{l}\text {-Aerodynamics losses estimation } \\
\text {-Analytical equations }\end{array}$ & $\begin{array}{l}\text {-Empirical relations } \\
\text {-Analytical and empirical equation } \\
\text { with wind tunnel data correction }\end{array}$ & $\begin{array}{l}\text { —Finite difference modeling } \\
\text { —Aerodynamics shape optimization }\end{array}$ \\
\hline $\begin{array}{l}\text { Geometry, weight } \\
\& \text { structure }\end{array}$ & $\begin{array}{l}\text {-General concept, structural coefficients } \\
\text { estimation based on available } \\
\text { technologies }\end{array}$ & $\begin{array}{l}\text { - Configuration definition and } \\
\text { arrangement of major subsystems } \\
\text { - Point mass calculation based } \\
\text { on statistical data }\end{array}$ & $\begin{array}{l}\text { - Detailed component design and mass } \\
\text { properties calculation } \\
\text { — Several loading condition consideration } \\
\text { —-Detailed statistical data }\end{array}$ \\
\hline Trajectory & $\begin{array}{l}\text {-Rocket equations and Keplers relationship } \\
\text {-Analytical staging optimization including } \\
\text { gravitational losses and numerical } \\
\text { simulation correction }\end{array}$ & $\begin{array}{l}\text {-2 and } 3 \text { DoF simulation } \\
\text { - Parametric trajectory optimization } \\
\text { - Control loss calculation }\end{array}$ & $\begin{array}{l}\text {-6 DoF POST (Program to Optimize } \\
\text { Simulated Trajectories) }\end{array}$ \\
\hline
\end{tabular}

The Multi-LoF MDO concept develops system design parameters sequentially and step-by-step as in previous conventional manual procedures. When the design procedure is broken down into several steps from simple to complex, logical and technical difficulties are solved chronologically.

From the computational viewpoint, initial design point selection or achievement is the critical point of optimization because an appropriate initial point may reduce optimization iterations. If the initial point is selected near the optimum point, the optimization iterations are reduced exponentially. In particular for high-LoF MDO problems, objective function computations and constraints evaluation can take several minutes to hours. The Multi-LoF MDO structure develops suitable initial design point and reduces computational expense. ${ }^{21)}$

\subsection{Implementation}

This paper implements two LoF structures. In each MDO structure (low LoF or medium LoF), the optimum SSPLV design refers to contributions from individual discipline optimization and integration of discipline optimization using equation as follows:

$$
\Delta_{\text {SSPLV Design }}=\left(\sum_{i} \Delta_{\text {Discipline } i}\right)+\Delta_{\mathrm{MDO}}
$$

The parameters of various disciplines such as aerodynamics, geometry, weight, structure, propulsion and trajectory were selected as design vector. Table 1 shows the disciplines LoF in the SSPLV conceptual design phase. The effects of four disciplines considered in the design process of SSPLV are not identical. Therefore, the appropriate level of fidelity for each MDO structure is defined. The low LoF MDO structure named CONceptual DESign (CONDES) integrates all disciplines in a single code and generates a feasible initial design point by using analytically optimized design methods considering mission requirements and main technological constraints. ${ }^{22)}$ It can also scan a wide range of design space. A feasible initial design point near the optimum greatly reduces the computational load of optimization. In the medium
LoF structure, the four disciplinary codes have a higher order, accuracy and level of detail than the low LoF MDO structure. The solid propellant motor design code plays the main role in the design structure matrix of the vehicle. A relatively high LoF motor design code is developed. This tool named Solid Propellant Rocket Motor Design, Analysis, Simulation and Optimization (SPRM DASO) consists of many modules, such as Solid Rocket Motor (SRM) configuration design, internal ballistics simulation, nozzle design, motor structure design, insulation design, igniter design, etc. The SPRM DASO code allows design, analysis, simulation and optimization of the motor for each stage.

The aerodynamic code called AEROdynamics DESign (AERODES) is developed to calculate aerodynamic coefficients depending on Mach number and angle of attack and also performs fairing shape optimization. A Semi-empirical formula is used for the aerodynamic calculations. A disciplinary optimization procedure is developed to determine the optimum geometry of the fairing based on the second-stage diameter.

A quasi-3 DoF trajectory simulation code called TRAJECT is developed to calculate and optimize trajectory parameters and evaluate constraints. A Parametric optimized pitch program is inserted in TRAJECT. A structure design and analysis module called STRUCT is dedicated to design of miscellaneous elements of the structure, such as tail, stabilizers, interstages, etc. The Optimizer of medium LoF is selected based on sequential convex programming and the method of center algorithms, which are available in software called ADS. ${ }^{23)}$

\section{Problem Definition}

\subsection{Baseline configuration}

Figure 2 shows the baseline launch vehicle configuration in this study. The vehicle is divided into a two-stage vehicle and orbital module. The orbital module has an Orbital Maneuvering Vehicle (OMV). The external geometry and 


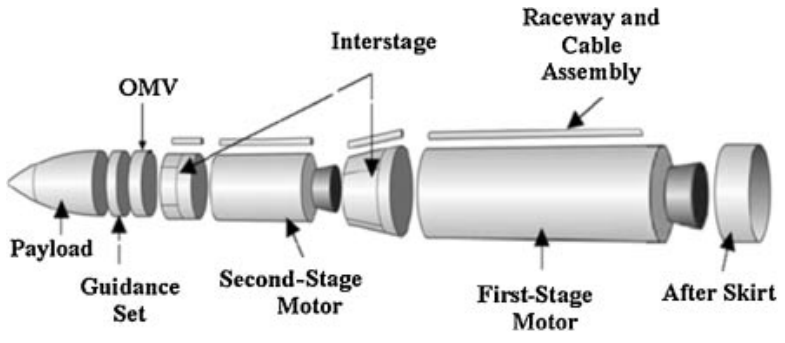

Fig. 2. Typical launch vehicle configuration.

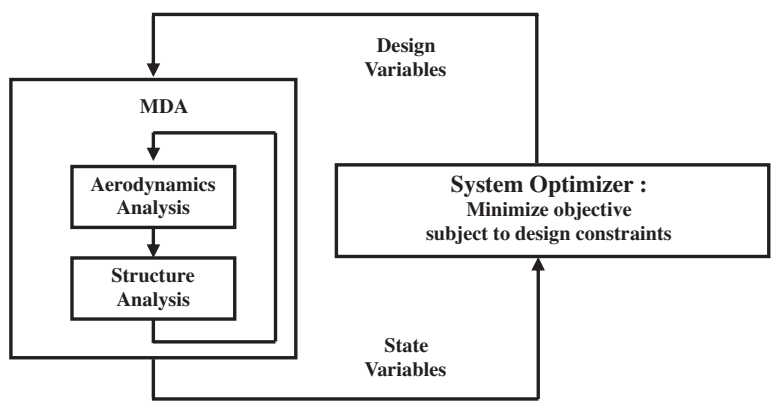

Fig. 3. MDF formulation.

internal packaging layout is determined for a reference vehicle. Change in diameters of the stages obviously changes the length of the vehicle. The fairing shape optimization is provided by AERODES module depending on the second-stage diameter.

\subsection{Performance index}

The concept of optimal design has different meanings for different design groups. In launch vehicle design, minimum weight concepts have been traditionally sought. In some cases, liftoff gross weight is selected as the minimization objective function. ${ }^{24,25}$ However, realizing that propellant is relatively inexpensive, most concepts have been designed to minimize dry weight. ${ }^{26-31)}$ Since it is often claimed that vehicle development costs tend to vary as a function of dry weight, this minimum dry weight vehicle may be considered a minimum development cost concept. However, as demonstrated by Brown et al. ${ }^{32}$ ) such an assertion is not rigorously true even when a weight-based cost model is used. Since the SSPLV propellant cost is considerable, minimization of SSPLV launch weight was chosen as the system performance index (objective function) for design optimization in this study.

\subsection{Problem formulation}

A MDO method for a given problem consists of a MDO formulation and an optimization algorithm. The former deals with problem decomposition, and mathematical issues, such as equivalence to the original problem and to alternative formulations are relevant. The latter deals with the solution procedures applied to the MDO formulation, and the properties of optimization algorithms as applied to the formulation are of interest.

A coupled aerodynamics-structure problem was used to illustrate two formulations that have been implemented in this work. The most obvious formulation is to stay with

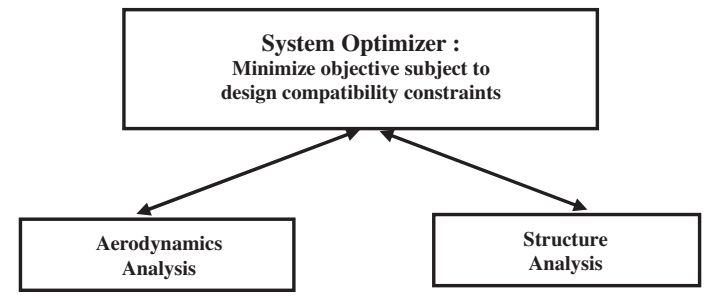

Fig. 4. IDF formulation.

the original problem and wrap an optimizer around it (Fig. 3). Using this MDF approach at each optimization cycle, the current trial point is a consistent solution of the MDA problem that requires iterations (usually of fixedpoint type) through all the disciplinary codes. The use of the word "feasible" in the name of the MDF formulation may be misleading. It is feasible only in the sense that the multi-disciplinary analysis may still represent a point that is infeasible with respect to the optimization problem where some constraints are violated.

However, the MDF formulation is not the only approach. Figure 4 shows the IDF formulation. Each IDF optimization cycle requires feasible solutions for each discipline analysis but not for the full multi-disciplinary analysis. The disciplinary coupling variables become a part of the design variable set in IDF, and compatibility constraints are added at the system level; consistent multi-disciplinary analysis is guaranteed only at convergence of the optimization.

This study used IDF and MDF formulations. The lowLoF MDO structure called CONDES has IDF formulation, and the medium LoF code uses MDF. Figure 5 shows this structure, the disciplines and data flow. A set of values is inserted into the input variables and the disciplines are run sequentially. Every analysis module not only calculates the intermediate variable and passes them to other disciplines, but also computes function values of the equality and inequality constraints that should be satisfied. Every iteration cycle for low-LoF lasts 20 seconds while the time for every iteration of the medium-LoF is about 16 minutes.

\subsection{Technical data and technological constraints}

Discipline input parameters that do not change during design cycle are considered as technical data. Technological constraints are requirements for technologies to be used. In other words, available technologies apply some limitation on design. Maximum and minimum values of rocket motor length, diameter, case strength and propellant loading density are some examples of technological constrains. Material specifications and propellant characteristics are known as technical data.

\subsection{Mission requirements}

Mission requirements consist of payload mass to be inserted into a given main mission orbit, injection accuracy, limitation on loads encountered by satellite, operational constraints such as range safety and launch azimuth.

\subsection{Design and interdisciplinary variables}

Table 2 lists selected design variables classified into three types. The types represent geometric shapes of the vehicles 
(diameter of each stage), propulsion performance (thrust and burning time of SRM's) and parameters of optimal flight trajectory (maximum angle of attack during first stage maneuver, final pitch angle and course time) respectively. CONDES passes the geometric and propulsion parameters to the medium-LoF MDO structure (design variables from $\mathrm{X} 1$ to X6 in Table 2).

The general layout of interdisciplinary data flow of the medium-LoF MDO is defined in Table 3. An integrated uniform design environment increases computational efficiency. All disciplinary codes have a design-oriented (batch run) version and a data dictionary allowing communication of all input and output variables without human interaction.

\section{Analysis Codes}

\subsection{Low-LoF conceptual design}

In conceptual design code, the mass distribution of a twostage solid propellant launch vehicle is evaluated so that the

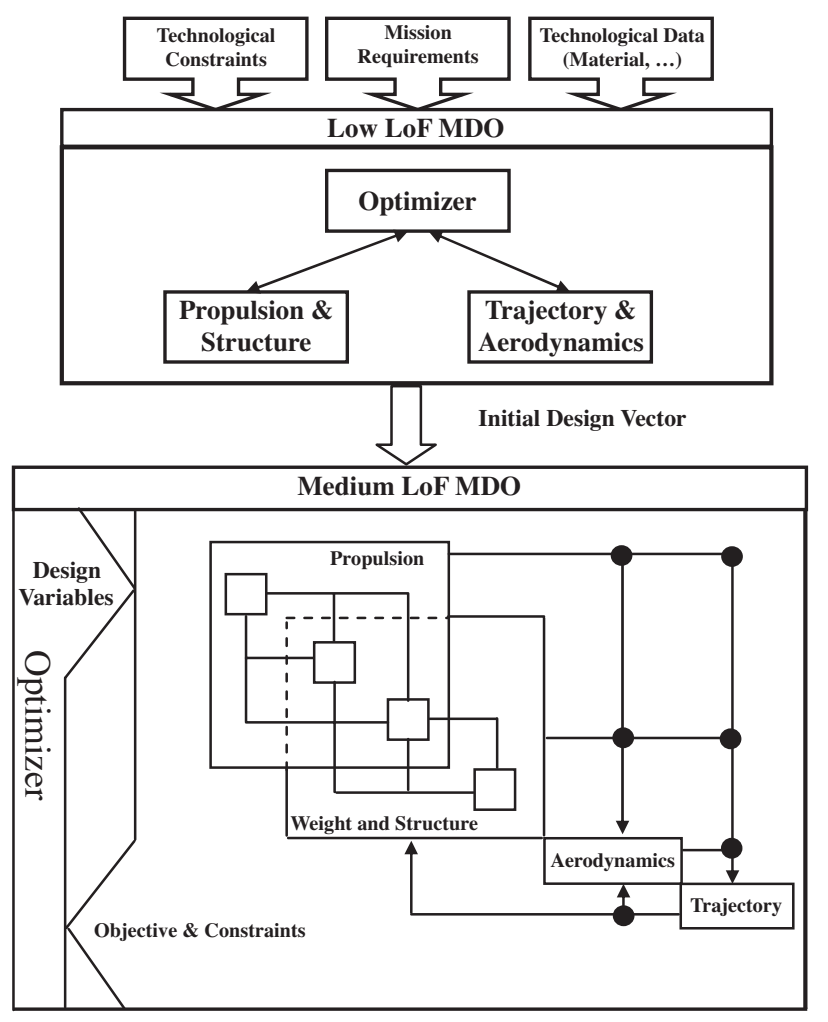

Fig. 5. Problem definition. ratio of total mass of the vehicle with respect to the payload mass remains minimum. ${ }^{22)}$ The following equations are supposed:

$$
\begin{aligned}
& m_{i}=m_{\mathrm{eng}_{i}}+m_{\mathrm{f}_{i}} \\
& m_{i}=\frac{m_{\mathrm{P}_{i}}\left(1-e^{\xi_{i}}\right)}{\frac{\alpha_{i}}{1+\alpha_{i}} e^{\xi_{i}}-1}
\end{aligned}
$$

where:

$$
\xi_{i}=\frac{V_{i}}{g I_{\mathrm{sp}_{V_{i}}}}, \quad \alpha_{i}=\frac{m_{\mathrm{eng}_{i}}}{m_{\mathrm{f}_{i}}}
$$

Thus, for the two-stage SSPLV, the ratio of the total mass to the payload mass may be found as follows:

$$
\frac{m_{\mathrm{stg}_{1}}}{m_{\mathrm{P}}}=\frac{1}{\left[\left(1+\alpha_{1}\right) e^{-\xi_{1}}-\alpha_{1}\right]\left[\left(1+\alpha_{2}\right) e^{-\xi_{2}}-\alpha_{2}\right]}
$$

Hence the design problem may be modeled as an optimization problem with the following objective function:

$$
F\left(\xi_{1}, \xi_{2}\right)=\left[\left(1+\alpha_{1}\right) e^{-\xi_{1}}-\alpha_{1}\right]\left[\left(1+\alpha_{2}\right) e^{-\xi_{2}}-\alpha_{2}\right]
$$

Also design variables of this constrained optimization problem are $\xi_{1}, \xi_{2}$, and the constraint equation is:

$$
V_{1}+V_{2}=V_{\mathrm{t}}
$$

Actual burnout velocity of the SSPLV may be found as

\begin{tabular}{|c|c|c|c|}
\hline \multirow{2}{*}{$\begin{array}{l}\text { Design variables } \\
\quad \text { (unit) }\end{array}$} & \multirow{2}{*}{ Descriptions } & \multicolumn{2}{|c|}{ Values } \\
\hline & & Min. & Max. \\
\hline $\mathrm{X} 1(\mathrm{~m})$ & First-stage diameter & 1.3 & 2.5 \\
\hline $\mathrm{X} 2(\mathrm{~m})$ & Second-stage diameter & 1.3 & 2.5 \\
\hline $\mathrm{X} 3(\mathrm{kN})$ & First-stage thrust & 1,500 & 1,700 \\
\hline $\mathrm{X} 4(\mathrm{kN})$ & Second-stage thrust & 150 & 300 \\
\hline X5 (s) & First-stage burning time & 60 & 90 \\
\hline X6 (s) & Second-stage burning time & 100 & 170 \\
\hline X7 (deg) & $\begin{array}{l}\text { Maximum angle of attack } \\
\text { during first-stage maneuver }\end{array}$ & 0 & 8 \\
\hline X8 (deg) & Final pitch angle & -30 & 15 \\
\hline X9 (s) & Course time & 0 & 30 \\
\hline
\end{tabular}
follows:

$$
V_{\mathrm{t}}=V_{\mathrm{t}}^{\prime}+V_{\mathrm{Loss}}
$$

where:

Table 2. Design variables.

Table 3. Interdisciplinary variables.

\begin{tabular}{lll}
\hline \multicolumn{1}{c}{ Outputs } & \multicolumn{1}{c}{ Inputs } & \multicolumn{1}{c}{ Parameters } \\
\hline Propulsion & Trajectory & Thrust, propellant mass, nozzle area and exit pressure etc. \\
Propulsion & Aerodynamics & Rocket motor lengths and diameter, etc. \\
Structure & Trajectory & Weights of rocket motor case, nozzle, inter-stage, etc. \\
Structure & Aerodynamics & Orbital module geometry and inter-stage length, etc. \\
Aerodynamics & Trajectory & Aerodynamics coefficients, pressure center, etc. \\
Trajectory & Aerodynamics & Mach number, Angle of attack, Reynolds number, Stage number \\
Trajectory & Structure & LV Mass, cross-section loading condition \\
\hline
\end{tabular}




$$
V_{\mathrm{t}}^{\prime}=V_{\text {Perigee }}=\left[\frac{\mu}{a}\left(\frac{1+e}{1-e}\right)\right]^{1 / 2}
$$

The proposed procedure to find the optimum mass distribution is as follows:

Step-1 $V_{\text {Loss }}$ is assumed zero for the first guess.

Step-2 An equal value is assumed as the first guess for $V_{1}$ and $V_{2}$.

Step-3 $\quad m_{1}$ and $m_{2}$ is calculated.

Step-4 $V_{\mathrm{t}}^{\prime}$ is calculated.

Step-5 $V_{\mathrm{t}}$ is calculated.

Step-6 Optimizing the objective function, the new values for $V_{1}$ and $V_{2}$ may be found.

Step-7 The new values for mass distribution between the stages may be found.

Step-8 Simulating the flight, a better approximation for velocity loss is calculated.

Step-9 Repeat steps 3 through 7 until convergence of Results.

CONDES benefits from a 2-DoF trajectory simulation to achieve velocity loss estimation. The following equation is used to evaluate the propellant mass for both stages:

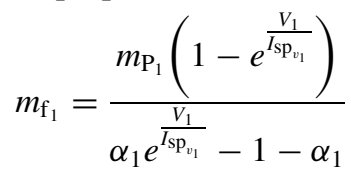

The velocity loss is calculated as follows:

$$
\begin{aligned}
V_{\mathrm{Loss}}= & \Delta V_{\mathrm{aer}_{1}}+\Delta V_{\mathrm{g}_{1}}+\Delta V_{\mathrm{eng}_{1}}+\Delta V_{\mathrm{c}_{1}} \\
& +\Delta V_{\mathrm{aer}_{2}}+\Delta V_{\mathrm{g}_{2}}+\Delta V_{\mathrm{eng}_{2}}+\Delta V_{\mathrm{c}_{2}}
\end{aligned}
$$

where:

$$
\begin{aligned}
& \Delta V_{\mathrm{g}}=g \int_{t_{1}}^{t_{2}} \sin \varphi \mathrm{d} t \\
& \Delta V_{\mathrm{c}}=\int_{t_{1}}^{t_{2}} \frac{F_{\mathrm{eng}}}{m}(1-\cos \sigma) \mathrm{d} t \\
& \Delta V_{\mathrm{eng}}=\int_{t_{1}}^{t_{2}} \frac{A_{\mathrm{e}} P_{\mathrm{h}}}{m} \mathrm{~d} t \\
& \Delta V_{\mathrm{aer}}=\int_{t_{1}}^{t_{2}} \frac{F_{\text {aer }}}{m} \mathrm{~d} t
\end{aligned}
$$

\subsection{Propulsion code}

The SPRM DASO code is developed to cover the whole SRM design space. All valuable conflicting inter-disciplinary parameters are considered. Using these modules in launch vehicle conceptual design phase improves design LoF and knowledge about design.

The code consists of six design modules, three analysis modules and one simulation module. The six design modules are motor configuration design, optimum nozzle profile design, grain design, case design, insulation and igniter design. The three analysis modules are nozzle performance analysis, grain regression analysis, and case and grain structure analysis. A one-dimensional internal ballistic module simulates the motor behavior, presents the thrust profile and grows the LoF for conceptual design phase. All modules derive design vector to optimize SRM. ${ }^{33)}$

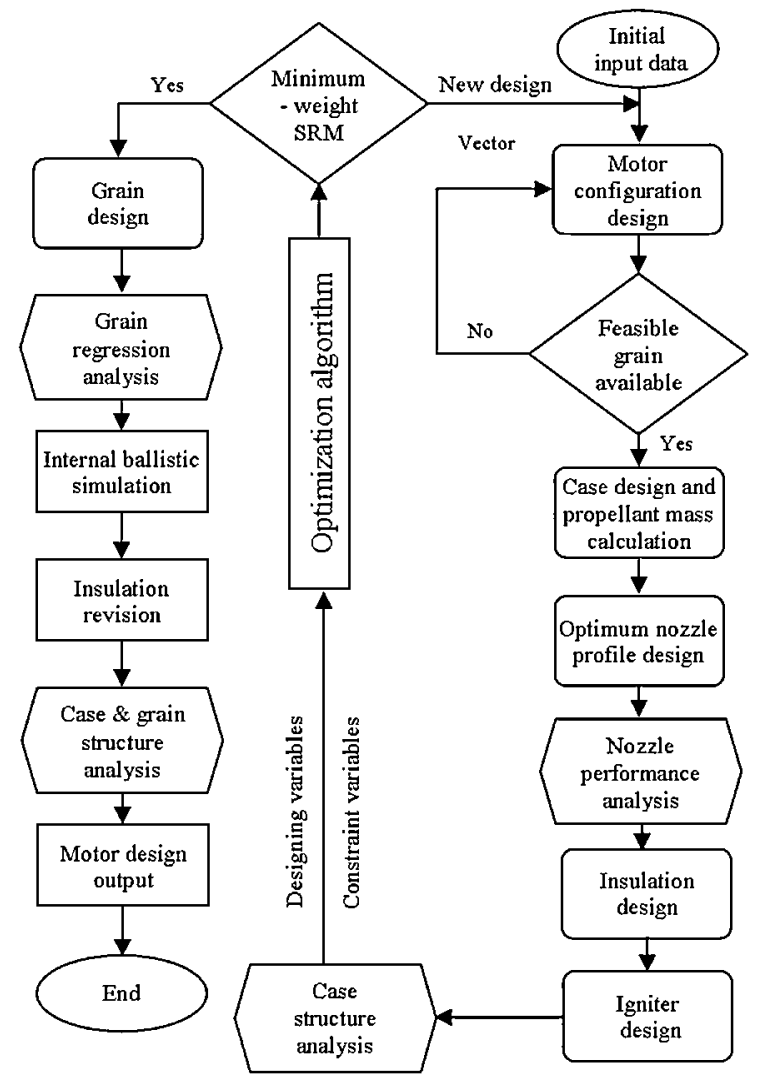

Fig. 6. SPRM DASO code procedure.

Figure 6 shows the SPRM DASO code procedure. There is a design loop and analysis procedure as illustrated. Input data is set when the problem is formulated. Invariable inputs and design variables are the two categories of input data used in this discipline.

The design optimization loop starts with motor configuration design to derive the main design parameter for the SRM. Computation of SRM geometrical and performance parameters is the main task of the motor configuration design module as shown in the first calculation block of Fig. 6. It uses a closed form of eleven analytical propulsion equations to generate a baseline configuration.

To reduce the design cycle, a special procedure was developed to show that the feasible grain is available. All feasible grain volumetric fractions with respect to web fractions are calculated by using the grain design module, and the available grain region is determined.

The SRM structure is composed of nozzle, closures and cylindrical section of case. The thickness of each section is calculated, and weight computed. Design is performed using the pressure vessel code. The weight and structure code calculate weight of other assemblies. Calculation details are presented in section 4.3. Propellant mass in the aft and end closures may be added to the grain mass in the cylindrical section.

At SRM nozzle design for SSPLVs, the main objective is maximizing thrust while minimizing nozzle weight. A conical and parabolic nozzle could be designed and analyzed by the optimum nozzle profile design module. In most research 
related to optimum nozzle profile, a generic parametric shape is taken into account.

There are two major functions in the insulation module selection of material and thickness design. Most current insulators for SRMs function as heat barriers primarily through ablation. The ablative insulation function divides the material into three zones: virgin-material zone, decomposition zone, and char zone. The interfaces separating these zones are indistinct, but even so, the zones can be defined by the principal phenomena found in each. The insulation module assumes available technologies and calculates the thickness of each zone.

A major igniter function is to increase the motor pressure to the level that the propellant can continue burning uniformly. Therefore, in designing a solid propellant rocket igniter, the pressure caused by igniter operation should be taken into account. In this regards, the energy released and propellant weight must be determined. This module also computes dimensions and weight of igniter.

The design variables, constraint values and SRM weight are applied to the optimization module. If the optimization criteria don't match, a new design vector is sent to the next loop. When optimum SRM is achieved, the grain design and optimization are performed.

Grain design for a SRM necessitates compromises between conflicting requirements of ballistic performance, structural integrity, mission reliability and geometric constraints. Consequently, there must be a coordinated exchange of information among other modules. In this regard, several design requirements are imposed on grain design and optimization. Tolerance of propellant mass, initial port area and average burning area must be less than the maximum tolerance of allowable values. Also neutrality and volumetric loading fraction have limitations. Minimization of sliver is the objective function of grain design module. The grain design module models two types of star grains, such as convex and concave. The star grain design space is not continuous. Thus, the parametric optimization method was used to derive the best available star grain configuration.

Analytical formulation is used to analyze grain regression. One-dimensional ballistic simulation is performed in three temperature conditions. Internal insulation is revised based on the critical condition. Finally, case and grain structure analysis is performed in three load conditions.

Unsteady quasi one-dimensional flow simulation is performed by internal ballistics simulation module. This module considers pressure-dependent burning rate and several impulse losses, including boundary layer, divergence losses and two-phase flow efficiency.

The study of propellant structure, its response to loads and its resistance to failures are vital parts of feasible SRM. From the time that a solid propellant rocket grain is cast until it burns during its mission, it is subjected to an array of stress-inducing environments such as continuing forces of gravity, loads from propellant curing and temperature excursion, shocks and vibrations due to handling and captive flight, and finally the pressurization and accelerations that accompany ignition, launch, and flight. These loads cause displacements, strains, and stresses. In this module, three types of loads are considered; thermal shrinkage, internal pressure and axial acceleration. Case structural analysis was performed again with revised pressure, and the safety factor was checked.

\subsection{Weight and structure code}

The weight and structure code consists of two parts. The first is structural design and weight estimation, and the second is structural analysis. As mentioned, SRM case design and weight estimation are accomplished in the propulsion code.

The structural design procedure includes thickness calculation of miscellaneous parts of the structure under various loading conditions. Loading conditions are classified into two phases of pre-flight loading and aft-flight loading. ${ }^{34)}$ The pre-flight loading contains the transportation, erection and pre-launch situations. The aft-flight loading contains the active and passive flight profile. In this procedure, the diagrams of axial, bending and shearing loads are obtained subject to the mentioned loading conditions. The equivalent axial load technique is used as follows:

$$
F_{\text {eq }}=F_{x}+2 \frac{M}{a}-\pi P a^{2}
$$

Using the severest equivalent axial load, the thickness of various parts of the structure is calculated considering the buckling and yield stress criteria. Solving the stability equations of a cylindrical shell, assuming simply supported boundary conditions, the theoretical axial buckling load is found as follows:

$$
\frac{P_{\mathrm{cr}}}{2 \pi a}=\frac{E^{h^{2}} / a}{\left[3\left(1-v^{2}\right)\right]^{1 / 2}}
$$

For compatibility of theoretical and experimental results, the following correction factor was used:

$$
K=k^{\prime} k_{\mathrm{M}}
$$

where:

$$
\begin{aligned}
& k^{\prime}=0.605-0.505\left[1-\exp \left(-\frac{1}{16} \sqrt{\frac{a}{h}}\right)\right] \\
& k_{\mathrm{M}}=1+1.25\left(\frac{2 M}{F_{x} a}\right) /\left(1+\frac{2 M}{F_{x} a}\right)
\end{aligned}
$$

The critical buckling load using the correction factor above is found as follows:

$$
\frac{p_{\mathrm{cr}}}{2 \pi a}=K \frac{E^{h^{2}} / a}{\left[3\left(1-v^{2}\right)\right]^{1 / 2}}
$$

The correction factor $K$ is presented by Balabokh. ${ }^{35)}$ Finally, the thicknesses of cylindrical parts of the SSPLV are found as follows:

$$
h=\left(\frac{f F_{\mathrm{eq}}}{2 \pi(0.605) K E}\right)^{1 / 2}
$$

To calculate flight loading, the TRAJECT simulation predicts the axial, shearing and moment loadings in all sections 


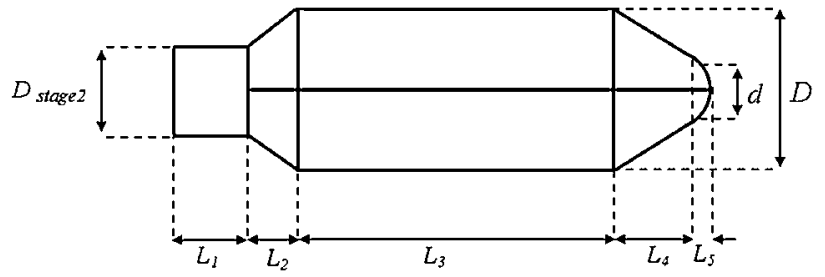

Fig. 7. Hammerhead fairing.

of the SSPLV. The thickness of cylindrical parts was calculated in the severest cross-section and loading conditions. Since the initial mass of the SSPLV is needed for flight simulation, predicted mass, which is found from the conceptual design procedure by CONDES, is considered as the first guess during simulation. The SSPLV mass after simulation may be used to improve the second simulation procedure. This process is repeated until the calculated mass of the parts converges. For conical parts of the structure, the same equation as above is used to evaluate the buckling load and thickness of elements, but because radius changes along the length of the truncated cone, the largest radius of the cone is assumed as the critical cross-section. Therefore, an equivalent cylinder with this radius can be used for calculating the buckling load of truncated conical parts.

\subsection{Aerodynamics}

The aerodynamic module has functions of aerodynamic calculation and fairing optimization. ${ }^{36)}$ Consequently, it includes two main routines: aerodynamic coefficients calculations, and optimization routines. For aerodynamic calculation, the geometry and flight conditions are used as input data, and the routine returns the aerodynamic characteristics of axial, normal, and pitching moment coefficients, center of pressure and static margin. Since the calculation of aerodynamic characteristic is a small portion of the main code, it should be accomplished in the minimum possible time with acceptable accuracy for a wide range of flight conditions, ranging from the subsonic to hypersonic regime. Based on the main code accuracy, MD, which is a well-known, semi-empirical aerodynamic tool, was used to calculate aerodynamic characteristics.

Fairing optimization is another task of the aerodynamic module where the hammerhead of the vehicle is designed. A SSPLV may be used as a suborbital test vehicle so the fairing could be a reentry vehicle. The objective function is minimum axial force under several constraints. These constraints are fairing length to base diameter ratio $(L / D)$, fairing nose diameter to base diameter ratio $(d / D)$, static margin and volume of the payload. The proposed configuration of the fairing is shown in Fig. 7. This configuration comprises a spherical blunt nose and two or three truncated cones. The optimization variables are the angle of cones and the axial location of cranks.

A non-gradient-based optimization technique is used to find the optimum point. The method is the so-called Direct Search Complex algorithm developed by Jones and Perttunen. ${ }^{37)}$ The name Direct for this algorithm is derived from one of its main features of dividing the $\mathrm{N}$-dimensional

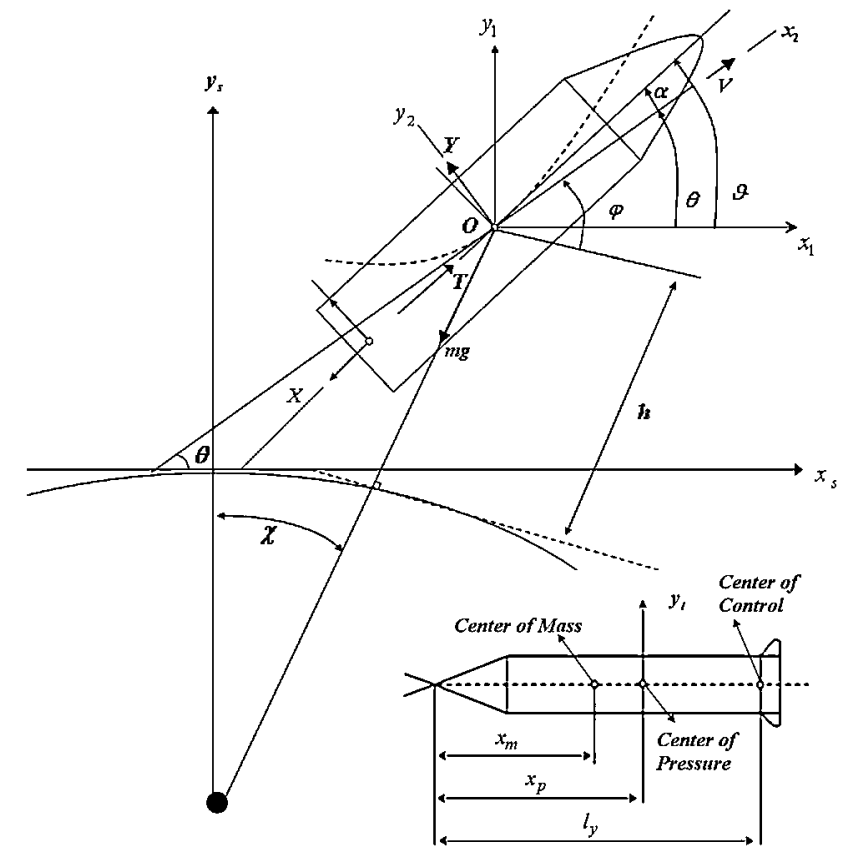

Fig. 8. Launch vehicle trajectory parameters.

search domain into sub-domains, where $N$ represents the number of design variables. The search is started inside the sub-domain in special manner and then sub-domains are divided again into smaller divides and the search process is continued inside the divided domains. This procedure is continued to reach the optimum point.

\subsection{Trajectory analysis}

This study uses a quasi-3 DOF trajectory analysis. ${ }^{38)}$ State variables are velocity, flight path angle, orbital velocity, altitude and mass. The following motion equations are used as modeled in Fig. 8:

$\begin{aligned} \dot{V}= & \frac{1}{m}\left[\left(T-X_{\mathrm{c}}-X_{\mathrm{aer}}\right) \cos \alpha-Y_{\mathrm{aer}} \frac{l_{y}-x_{\mathrm{p}}}{l_{y}-x_{\mathrm{m}}} \sin \alpha\right] \\ & -g \sin \varphi\end{aligned}$

$\dot{\varphi}=\frac{1}{m V}\left[\left(T-X_{\mathrm{c}}-X_{\mathrm{aer}}\right) \sin \alpha\right.$

$$
\left.-Y_{\text {aer }} \frac{l_{y}-x_{\mathrm{p}}}{l_{y}-x_{\mathrm{m}}} \cos \alpha\right]-\left(\frac{g}{V}-\frac{V}{r}\right) \cos \varphi
$$

where:

$$
\begin{aligned}
& \alpha=\vartheta-\varphi+\chi \\
& \dot{r}=V \sin \varphi \\
& \dot{\chi}=\frac{V}{r} \cos \varphi
\end{aligned}
$$

The parametric profile for the optimal pitch trajectory was selected by applying Pontriyagin's maximum principle and constraints on the motion of the first stage. ${ }^{39)}$

The result of launch trajectory design formulates an optimal pitch program with three free parameters. These parameters are the maximum angle of attack during the first maneuver of the launch vehicle, the pitch angle of the second stage and the course time. Since the selected trajectory parameters play important roles in the design cycle, they 


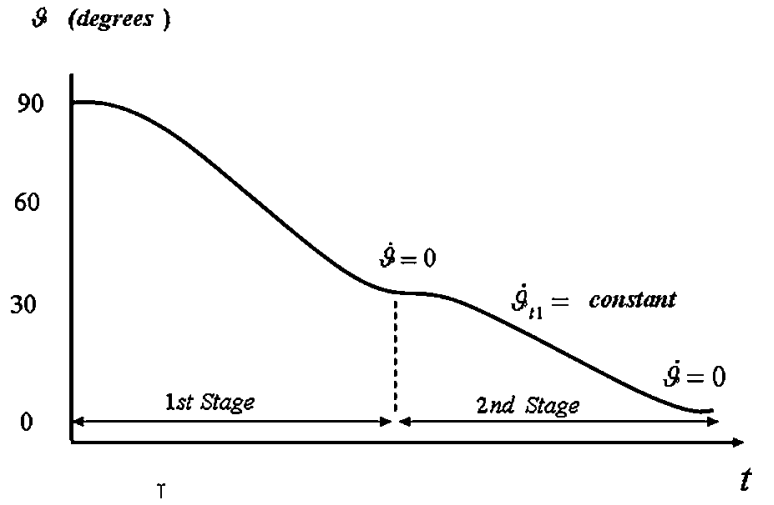

Fig. 9. Typical optimized pitch program profile.

Table 4. Some important inputs.

\begin{tabular}{lll}
\hline & \multicolumn{1}{c}{ Inputs } & \multicolumn{1}{c}{ Values } \\
\hline Structure & Density & $7,830 \mathrm{~kg} / \mathrm{m}^{3}$ \\
& Modulus of elasticity & $200 \mathrm{GPa}$ \\
& Ultimate stress & $1.5 \mathrm{GPa}$ \\
\hline Propellant & Density & $1,840 \mathrm{~kg} / \mathrm{m}^{3}$ \\
& Burning rate coefficient & $0.415 \mathrm{~cm} / \mathrm{s} / \mathrm{MPa}$ \\
& Burning rate exponent & 0.31 \\
& Loading density & $<92 \%$ \\
\hline Propulsion & Maximum length & $10 \mathrm{~m}$ \\
system & Maximum diameter & $2.5 \mathrm{~m}$ \\
geometry & & \\
\hline
\end{tabular}

have been selected as the system level design variables and optimized in the outer loop of the optimization as shown in Fig. 5.

The direct injection approach is selected, and the orbital maneuver and trim are performed by OMV. Figure 9 shows a typical optimal pitch program profile for a two-stage SSPLV.

\section{Optimization Problem and Solutions}

\subsection{Mission requirements and design constraints}

The SSPLV design reference mission for this study was to reach a $250-\mathrm{km}$ circular orbit and 34 degrees inclination with a $500 \mathrm{~kg}$ OMV. The launch site latitude was 34 degrees with eastward launch azimuth.

The stage separation height should be controlled between 30 and $50 \mathrm{~km}$. The launch site border limit causes the second-stage impact point to be less than $800 \mathrm{~km}$. The SSPLV length over diameter $(L / D)$ was limited from 8 to 13 .

\subsection{Technical data and technological constraints}

The multi-LoF formulation gives almost 350 input parameters and execution conditions. Some important technical data and technological constraints are shown in Table 4.

\subsection{Solutions}

Two optimization algorithms, such as Sequential Convex Programming (SCP) and Method of Centers (MoC), were

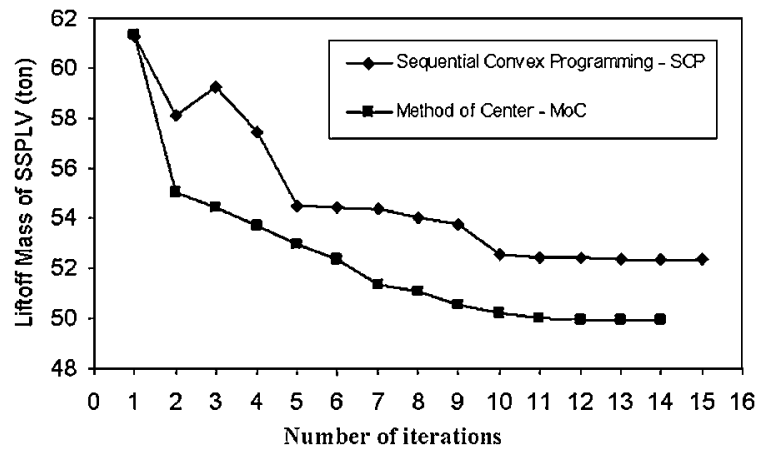

Fig. 10. Launch vehicle mass history-result of medium-LoF SSPLV MDO.

Table 5. Design variables of the medium LoF MDO.

\begin{tabular}{llc}
\hline & $\begin{array}{l}\text { Design } \\
\text { variables }\end{array}$ & Values \\
\hline First-stage diameter & X1 (m) & 1.9 \\
Second-stage diameter & X2 (m) & 1.5 \\
First-stage thrust & X3 (kN) & 1,600 \\
Second-stage thrust & X4 (kN) & 200 \\
First-stage burning time & X5 (s) & 75 \\
Second-stage burning time & X6 (s) & 130 \\
Maximum first-stage maneuver & X7 (deg) & 5 \\
Final pitch angle & X8 (deg) & -2 \\
Course time & X9 (s) & 19 \\
\hline
\end{tabular}

used in the optimizer of the medium-LoF MDO structure (Fig. 5). The results are shown in Fig. 10.

The initial point passed from the low-LoF code to the medium-LoF (X1-X6) and the initial values of the medium-LoF design variables $(\mathrm{X} 7-\mathrm{X} 9)$ are shown in Table 5.

The nonlinear design space causes many local optimums in SSPLV design problems. The MoC algorithm has acceptable performance to bypass local optimums and gives better results. The result of the implemented medium-LoF MDO shows that the SSPLV liftoff mass is decreased $12 \%$ to $19 \%$ as shown in Fig. 10.

The initial design point data and derived optimal solutions are listed in Table 6.

\section{Conclusion}

The MDO approach has a significant effect on design methodology. In design of next-generation space-launch systems, the MDO process faces new challenges.

Improving the conceptual design capability increases the fidelity of empirical disciplinary models, improves design solutions and insight by implementing design of experiments and optimization methods, and reduces workload and design cycle time through advanced frameworks. This automated procedure can identify feasible designs, calculate customer metrics, rank concepts, and present comparison and sensitivity data produced during optimization. 
Table 6. Initial point and optimal solution results.

\begin{tabular}{|c|c|c|c|c|}
\hline Parameters & & & $\begin{array}{r}\text { Initial } \\
\text { point }\end{array}$ & $\begin{array}{l}\text { Optimal } \\
\text { solution }\end{array}$ \\
\hline \multirow[t]{7}{*}{ First stage } & \multirow[t]{3}{*}{ Inputs } & Diameter (m) & 1.9 & 2 \\
\hline & & Burning time (s) & 75 & 60 \\
\hline & & Thrust (kN) & 1,600 & 1,500 \\
\hline & \multirow[t]{4}{*}{ Outputs } & $I_{\mathrm{sp}}:$ sea level (s) & 269 & 270 \\
\hline & & Propellant mass $(\mathrm{kg})$ & 44,480 & 32,221 \\
\hline & & Structure (kg) & 4,683 & 3,495 \\
\hline & & Length (m) & 11.5 & 8.3 \\
\hline \multirow[t]{7}{*}{ Second stage } & \multirow[t]{3}{*}{ Inputs } & Diameter $(\mathrm{m})$ & 1.5 & 1.7 \\
\hline & & Burning time (s) & 130 & 139.2 \\
\hline & & Thrust $(\mathrm{kN})$ & 200 & 273 \\
\hline & \multirow[t]{4}{*}{ Outputs } & $I_{\mathrm{sp}}:$ vacuum (s) & 297 & 296.2 \\
\hline & & Propellant mass $(\mathrm{kg})$ & 10,647 & 12,615 \\
\hline & & Structure $(\mathrm{kg})$ & 1,020 & 1,103 \\
\hline & & Length (m) & 3 & 4.4 \\
\hline
\end{tabular}

As the complexity and cost of launch vehicle increases, so the need for early systems analyses has significantly increased. The types of analyses discussed in this paper address only the conceptual and early preliminary design phases, where the major configuration and technology decisions dictate the largest percentage of total program costs. Advancing the capability for conceptual design to address the problems requires pursuing at least three major emphases considered here. First, improvements enhanced the fidelity of disciplinary engineering models and codes, especially in areas of empirical models such as estimations of weights and propulsion. Second, advances in computational methods search the design space and optimize the vehicle system toward selected, weighted objectives. Last, improvements in software frameworks automatically coordinate execution of coupled engineering disciplines (people and codes) to reduce workload and design cycle time.

Our approach has the potential to be used as a totally hands-off, multi-disciplinary conceptual design optimization. There is not user interaction or decisions during design and optimization; the user merely defines the goal and constraints on design variables.

The performance of the proposed structure is illustrated by implementation of two-levels of fidelity for a SSPLV. Then appropriate fidelity level for each discipline was developed. Every code was optimized at the discipline level and system level. The results show good performance in minimizing SSPLV launch weight.

\section{References}

1) Xianyu, W., Lin, X., Liang, J., Shibin, L., Xiaoqian, C. and Zhenguo, W.: The MDO Environment for Hypersonic Vehicle System Design and Optimization, AIAA Paper 2006-7119, 2006.

2) Zhao, Y., Chen, X. and Wang, Z.: SIDE: A Tool for Integrated Multidisciplinary Design Optimization of Spacecrafts, AIAA Paper 20067119, 2006.

3) Jian, G., et al.: A Distributed Multidisciplinary Design Optimization Architecture for Spacecraft Design, AIAA Paper 2002-5481, 2002.
4) McAllister, C. D., et al.: Multidisciplinary Design Optimization Testbed Based on Autonomous Under Water Vehicle Design, AIAA Paper 2002-5630, 2002.

5) Fitzgerald, C. J., et al.: A Conceptual Design Environment for Technology Selection and Performance Optimization for Torpedoes, AIAA Paper 2002-5590, 2002.

6) Ahlqvist, M. A., et al.: Designing and Optimizing Missiles in an Interactive Environment, AIAA Paper 2002-5624, 2002.

7) Zarda, P. R.: Multidisciplinary Design of Fire Control and Missile Systems Using Knowledge-Based Engineering Architecture, NIST ITEM 2000, 2000.

8) Tratabini, P. V., et al.: Multidisciplinary Analysis of a Lifting Body Launch Vehicle, J. Spacecraft Rockets, 39 (2002), pp. 788-795.

9) Unger, E. R., Hutchison, M. G., Rais-Rohani, M., Haftka, R. T. and Grossman, B.: Variable-Complexity Multidisciplinary Design of a Transport Wing, Int. J. System Automation: Research and Applications (SARA), 2 (1992), pp. 87-113.

10) Hutchison, M. G., Unger, E. R., Mason, W. M., Grossman, B. and Haftka, R. T.: Variable Complexity Aerodynamic Optimization of a High-Speed Civil Transport Wing, J. Aircraft, 31 (1994), pp. 110116 .

11) Kaufman, M.: Variable-Complexity Response Surface Approximations for Wing Structural Weight in HSCT Design, Master Thesis, Virginia Polytechnic Institute and State University, Blacksburg, VA, 1996.

12) Balabanov, V., Haftka, R. T., Grossman, B., Mason, W. H. and Watson, L. T.: Multidisciplinary Response Surface Model for HSCT Wing Bending Material Weight, AIAA Paper 98-4804, 1998.

13) Burgee, S. L., Giunta, A. A., Bablanov, V., Grossman, B., Mason, W. H., Narducci, R., Hafka, R. T. and Watson, L. T.: A Coarse Grained Parallel Variable-Complexity Multidisciplinary Optimization Paradigm, Int. J. Supercomputing, 10 (1996), pp. 269-299.

14) Giunta, A. A. and Watson, L. T.: Comparison of Approximation Modeling Techniques: Polynomial versus Interpolating Models, AIAA Paper 98-4758, 1998.

15) Knill, D. L., Giunta, A. A., Baker, C. A., Grossman, B., Mason, W. H., Haftka, R. T. and Watson, L. T.: Response Surface Models Combining Linear and Euler Aerodynamics for Supersonic Transport Design, J. Aircraft, 36 (1999), pp. 75-86.

16) Shibin, L., Wencai, L., Zhiyong, L. and Zhenguo, W.: Application of Taguchi Design Methods and Uniform Design Methods to Scramjet Propulsion System Optimization for Hypersonic Cruise Vehicle, AIAA Paper 2003-5193, 2003.

17) Turner, M. G., Reed, J. A., Ryder, R. and Veres, J. P.: Multi-Fidelity Simulation of a Turbofan Engine with Results Zoomed into MiniMaps for a Zero-D Cycle Simulation, NASA TM 2004-213076, 2004.

18) Kroo, I.: Multidisciplinary Optimization Applications in Preliminary Design-Status and Directions, AIAA Paper 1997-1408, 1997.

19) Jodei, J., Ebrahimi, M. and Roshanian, J.: An Automated Approach to Multidisciplinary System Design Optimization of Small Solid Propellant Launch Vehicle, 1st International Symposium on Systems and Control in Aerospace and Astronautics, Harbin, China, 2006.

20) Roza, Z. C.: Simulation Fidelity Theory and Practice-A Unified Approach to Defining, Specifying and Measuring the Realism of Simulations, DUP Science, 2004.

21) Vukelich, S. R., et al: Missile DATCOM Volume 1, AFWALTR-863092, Vol-1, 1988.

22) Mirshams, M.: Low Level of Fidelity Conceptual Design of Small Solid Propellant Launch Vehicles, MDO Laboratory, Dept. of Aerospace Engineering, K. N. Toosi University of Technology, MDO-LABTR122, 2005 (in Persian).

23) Vanderplaats, G. N.: ADS-A Fortran Program for Automated Design Synthesis, NASA CR 177985, 1985.

24) Freeman, D.: Future Space Transportation System Study, Aerospace America, 21, June (1983), pp. 36-56.

25) Martin, J.: Orbit on Demand, Aerospace America, 23, Feb. (1985), pp. $46-48$

26) Freeman, D., Stanley, D., Camarda, C., Lepsch, R. and Cook, S.: Single-Stage-To-Orbit: A Step Closer, IAF Paper 94-V3.534, 1994.

27) Stanley, D., et al.: Rocket-Powered Single-Stage Vehicle Configuration Selection and Design, AIAA Paper 93-1053, 1993. 
28) Engelund, W., Stanley, D., McMillin, M. and Unal, R.: Aerodynamic Configuration Design Using Response Surface Methodology Analysis, AIAA Paper 93-3967, 1993.

29) Lepsch, R., Stanley, D. and Unal, R.: Dual-Fuel Propulsion in SingleStage Advanced Manned Launch System Vehicle, J. Spacecraft Rockets, 32 (1995), pp. 417-425.

30) Braun, R., Powell, R., Lepsch, R., Stanley, D. and Kroo, I.: Comparison of Two Multidisciplinary Optimization Strategies for Launch Vehicle Design, J. Spacecraft Rockets, 32 (1995), pp. 404-410.

31) Olds, J.: System Sensitivity Analysis Applied to the Conceptual Design of a Dual-Fuel Rocket SSTO, AIAA Paper 94-4339, 1994.

32) Brawn, R. D., Moore, A. A. and Kroo, I. M.: Use of Collaborative Optimization Architecture for Launch Vehicle Design, AIAA Paper 1996-4018, 1996.

33) Ebrahimi, R.: Solid Propellant Rocket Motor Design, Analysis, Simulation and Optimization (SPRM DASO) Code, MDO Laboratory, Dept. of Aerospace Engineering, K. N. Toosi University of Technology, MDO-LAB-TR140, 2005 (in Persian).
34) Mirshams, M.: Small Solid Propellant Launch Vehicles Structure Analysis and Weight Estimation, MDO Laboratory, Dept. of Aerospace Engineering, K. N. Toosi University of Technology, MDOLAB-TR128, 2005 (in Persian).

35) Balabokh, L. E., et al: Principles of Rocket Structures, Machinostroienie, Moscow, 1989 (in Russian).

36) Mirzaee, M.: Aerodynamics and Shape Optimization, MDO Laboratory, Dept. of Aerospace Engineering, K. N. Toosi University of Technology, MDO-LAB-TR129, 2005 (in Persian).

37) Jones, D. R., Perttunen, C. D. and Stuckmann, B. E.: Lipschitzain Optimization without the Lipschitz Constant, J. Optimi., Theory Appl., 79 (1993), pp. 157-181.

38) Roshanian, J.: Trajectory Simulation and Optimization, MDO Laboratory, Dept. of Aerospace Engineering, K. N. Toosi University of Technology, MDO-LAB-TR115, 2005 (in Persian).

39) Malyshev, V. V., et al.: Aerospace Vehicle Control, Modern Theory and Applications, FAPESP Publication, Sao Paolo, 1996. 